\title{
Role of shielding in modeling cryogenic deuterium pellet ablation
}

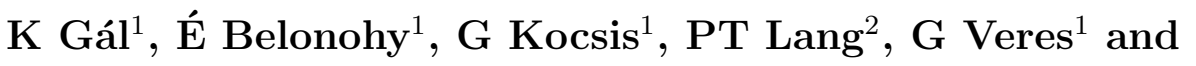 \\ ASDEX Upgrade Team ${ }^{2}$ \\ ${ }^{1}$ KFKI-Research Institute for Particle and Nuclear Physics of HAS, EURATOM \\ Association, P.O.Box 49, H-1525 Budapest-114, HUNGARY \\ ${ }^{2}$ Max-Planck-Institut für Plasmaphysik, EURATOM Association, Boltzmannstr. 2, \\ 85748 Garching, GERMANY \\ E-mail: gal@rmki.kfki.hu
}

\begin{abstract}
For the better characterization of pellet ablation, the numerical LLP code has been enhanced by combining two relevant shielding mechanism: that of the spherically expanding neutral cloud surrounding the pellet and of the field elongated ionized material forming a channel flow. In contrast to our expectations the presence of the channel flow can increase the ablation rate although it reduces the heat flux traveling through it. The contribution of the different shielding effects in the ablation process is analyzed for several pellet and plasma parameters and an ablation rate scaling is presented based on simple regression in the ASDEX Upgrade pellet and plasma parameter range. Finally the simulated results are compared to experimental data from typical ASDEX Upgrade discharges.
\end{abstract}

PACS numbers: 52.40.Hf, 52.55.Fa, 28.52.Cx

Submitted to: Nucl. Fusion 


\section{Introduction}

The refueling by cryogenic hydrogen isotope pellet injection in large tokamaks was proven to be the most promising way to compensate particle losses [1] providing access to operational regimes, that were unavailable by gas puffing [2]. Besides, plasmas with high confinement need to be controlled, as they are favorable for several instabilities such as Edge Localized Modes (ELMs). ELMs can also be triggered by pellets causing frequent particle and energy losses on a short time scale preventing the formation of large heat loads on the divertor due to the less frequent intrinsic ELMs [3]. The use of pellets for ELM mitigation purposes renewed the interest to better understand the ablation process of hydrogen isotope pellets injected into hot plasmas.

The interaction of hydrogen isotope pellet (in the following pellet) with the hot magnetized plasma is a complicated, time dependent and fully 3 dimensional magnetohydrodynamic phenomenon. This interaction can be described by the following simplified picture. Once a pellet enters the plasma the energy transported by the ambient plasma particles immediately start to ablate it. Considering the very low sublimation energy of the hydrogen isotope ice $\left(\epsilon_{\text {sub }} \sim 0.005 \mathrm{eV} /\right.$ particle $)$, the ablatant rapidly (on a 100ns time scale) forms a cloud around the pellet which shields the pellet completely from the ambient target plasma. Due to high dissociation $(\sim 4.5 \mathrm{eV})$ and ionization energy $(13.6 \mathrm{eV})$ of the neutral hydrogen near to the pellet surface the cloud consists of neutral particles. The energetic particles of the ambient target plasma heat the cloud by scattering processes therefore the cloud expands spherically. This is a self-regulating process: once the shielding is complete the ablation is stopped. As the cloud expands, the shielding is reduced restarting the ablation again and completing the shielding. This process converges to steady state. Further away from the pellet surface, the interaction of the neutrals with the background plasma particles will ionize the primarily neutral cloud. The partly ionized cloud expands perpendicular to the magnetic field and interacts with the field, therefore its cross-field motion is stopped and the expansion undergoes a channel flow along the magnetic field lines.

The ambient target plasma particles penetrating through the pellet cloud carry a current which gives rise to an electrostatic field driven current in the cloud. On the other hand, the ambient plasma particles in the cloud together with the resident thermal charge carriers set up an electrostatic double shield at the pellet cloud - ambient target plasma interface, repelling a part of the target plasma electrons, and slightly accelerating the ones entering the pellet cloud [4]. The electrostatic field in the cloud does not let the particles originating from the pellet leave the cloud, they interact in the cloud, thus the cloud loses energy through expansion, ionization and radiation composed of line radiation and Bremsstrahlung [5]. Also both the ablation and cloud formation extracts energy from the ambient plasma. The energy reservoir of the ambient target plasma is not unlimited: large pellets $\left(r_{p} \sim 1 \mathrm{~mm}\right.$, ie. the largest available pellet at ASDEX Upgrade) can cool it down considerably.

During its ablation the pellet leaves a part of its cloud attached to the magnetic field 
as it travels with a given velocity. Additionally the ionized pellet cloud itself can move across the magnetic field lines (e.g. by grad B drift or by cloud instabilities $[6,7,8]$ ).

To get the most complete theoretical description of the above complex phenomenon, a number of models using different approximations with different complexity have been developed in last the 30 years.

Among these models the Neutral Gas Shielding (NGS) model has become the most popular and widely used one [9]. It only considers a symmetric and spherically expanding neutral cloud which shields the pellet and uses a simple 1 dimensional geometry. The electrons of ambient target plasma are treated as monoenergetic and they heat the neutral cloud surrounding the pellet. The self-regulation of the ablation was taken into account presuming that the heat flux of the target plasma reaching the pellet surface is always zero. The hydrodynamic equations were solved by using a steady state assumption, and the $N^{\prime}$ ablation rate (the total number of ablated particles/second) was obtained as a power function of the plasma electron temperature $T_{e}[\mathrm{eV}]$, electron density $n_{e}\left[\mathrm{~m}^{-3}\right]$ and the pellet radius $r_{p}[\mathrm{~mm}][10]$ :

$N^{\prime}=3.78 \cdot 10^{16} \cdot r_{p}^{1.33} \cdot n_{e}^{0.33} \cdot T_{e}^{1.64}$

Here we would like to mention that SI units are used, except the temperature and energy which are given in $\mathrm{eV}$.

The NGS model was further developed to describe not only the spherically expanding cloud but also a field aligned flow of the cloud particles [10, 11, 12]. The electrostatic shielding was also adapted to this model [13].

Several attempts aimed at the 2D implementation of the pellet ablation and cloud expansion. A kinetic heat flux model taking into account the influence of magnetic field on the cloud heating and on the ablation process itself was developed [14]. The possibility of a shock wave formation due to ionization in connection to the strong pressure anisotropies in the pellet cloud and the melting of the pellet was studied as well $[14,15]$. Recent 2D MHD simulation [16] showed that the pressure anisotropy is less important when both the electrostatic shielding and the $j \times B$ forces are taken into account.

Already the early experiments [17, 18] showed that the pellet is surrounded by a cigar shaped cloud which is elongated along the field lines, thus L.L. Lengyel and his co-workers $[19,20]$ were focusing on understanding this observation. According to the experiments, a code consisting of threes main modules was developed. The first module of the code is an 1.5 dimensional time dependent magneto-hydrodynamic model which describes the cross-field expansion, deceleration and full stopping of the pellet cloud. Expansion in both direction is considered: the cross field expansion (1D) and field elongated expansion ( $0.5 \mathrm{D}$ as the pellet cloud is only discretized in the cross field direction). This module determines the radius of the channel $\left(r_{e f f}\right)$, the extension of the cigar shaped cloud perpendicular to the magnetic field lines where the field elongated expansion takes place. The second module is a multi Lagrangian cell, time dependent hydrodynamical code that describes the pellet ablation and cloud expansion 
as a 1 dimensional channel flow along the field lines. Finally the third module takes into account effects associated with the traversing motion of the pellets, particularly the periodic crossing of the ionization region created in front of the pellet. We will refer to this code as the LLP code.

The 1 dimensional channel flow code, the hydrodynamic part of the LLP code [20], takes care of the following processes in the cloud elongated along the magnetic field lines and calculates the ablation rate:

- penetration of the ambient target plasma particles into the cloud and their collisional energy transfer to the cloud particles,

- the electrostatic field in the cloud,

- the potential wall formed at the pellet cloud - ambient plasma interface,

- gas dynamic expansion along the field lines,

- the ionization state of the pellet cloud particles by finite rate atomic processes (electron impact ionization, radiative and three body recombination),

- radiation of the cloud particles,

- flux limited heat diffusion in the pellet cloud.

The assumed Maxwellian energy distribution of the ambient target plasma particles was modeled by a number of monoenergetic electron beams directed towards the pellet surface. The pellet ablates at a rate set by the balance between the heat flux reaching the pellet surface (the sum of the heat flux of the discrete energy groups) and the energy required to remove particles from the solid surface (sublimation energy):

$N^{\prime}=\frac{Q_{e}}{\epsilon_{\text {sub }}}$

The particle source in a channel persists during the so called residence time. The residence time in such a channel is the time necessary for the pellet to cross it, $t_{r e s}=2 * r_{e f f} / v_{p}$, where $v_{p}$ is the pellet velocity. After the pellet crosses this channel the particle source is shifted to the next location, where the whole process is repeated. When the pellet moves from one tube to another a part of the cloud travels with it. The magnitude of this pre-shielding is determined semi-empirically. The pellet moves from channel to channel until it is completely ablated.

The NGS model describes only the spherically expanding part of the cloud while the hydrodynamic part of the LLP model describes only the channel flow. A priori we do not know which one is the dominating cloud part because this depends on the pellet and plasma parameters and generally none of them are negligible. Moreover, due to the high density of the cloud near the pellet surface, this spherically expanding part probably plays a determining role in the shielding. On the other hand the knowledge of the parameters of the field aligned cloud part is also indispensable for the determination of the cloud drifts. Consequently a model which considers both the spherical expansion and the channel flow would give a more complex description of the pellet - plasma interaction. 
Our aim was to combine these two approaches to get a more general description of the pellet ablation. The combination of the NGS and LLP models was realized in a simple way and the results are reported in this paper. The name of this new code is the Hybrid code.

The paper is organized as follows: in section Section 2 we give a detailed description of the model. As we would like to emphasize the shielding effects of the spherically expanding neutral cloud and of the channel flow, their importance is pointed out in Section 3. By the use of a linear regression fit a simple formula for the ablation rate is given in Section 4. The results given by the code are compared to typical experiments in Section 5. Finally we will summarize (Section 6) the results presented in this paper.

\section{The model}

According to our physical picture, a neutral cloud forms almost instantaneously around the pellet as particles are extracted from the pellet surface [11] and it expands spherically with a velocity close to the sonic velocity. The density of this spherically expanding cloud drops dramatically with the distance from the pellet surface, its shielding effects becoming negligible at about $10 \cdot r_{p}[21]$. However at the same radius ionization sets in and the spherical expansion transforms into a channel flow [20]. The particle source of the channel flow region is the spherical cloud which feeds it with neutral atoms with temperatures of around $1 \mathrm{eV}$ traveling with the sonic velocity corresponding to this temperature.

The spherically expanding cloud part and the cloud part expanding along the field lines is handled separately. It is assumed that a neutral cloud is always present in the vicinity of the pellet, while the ionized cloud forms gradually, its effect becoming visible after a few microseconds. This way no semi-empirically determined pre-shielding had to be assumed as in the original LLP model. The ionized cloud part forming a channel flow is described by the hydrodynamic part of the LLP code. On the other hand the quasi-steady state spherical expansion of the neutral cloud is supposed to be sustained by the energetic particles of the ambient target plasma crossing the channel flow region, where their energy and flux are reduced by elastic and inelastic collisions. This way the pellet ablation can be described by the analytical solution of the NGS model using an equivalent ambient plasma temperature and density at the spherical cloud and channel flow interface.

To avoid confusion the following notations will be used. The pellet is injected into the target or background plasma. In the pellet cloud there are cold electrons of the cloud itself (see later) and hot electrons originating from the target plasma. Regarding toroidal expansion, the pellet is situated at $z=0$ (see Figure 1) and the cloud expands both in the positive and negative directions. As the pellet cloud is symmetric, only expansion in the positive direction is considered (such calculation are referred to as single side calculation). In the toroidal direction ( $z$ direction) the following surfaces are defined: The pellet surface (surface $1, z=z_{1}$ ) is the boundary of the solid hydrogen isotope 
ice. The spherical cloud surface (surface $2, z=z_{2}$ ) is the boundary of the spherically expanding neutral cloud and the channel flow. The whole pellet cloud (surface $4, z=z_{4}$ ) ends with an electrostatic double shielding (potential drop). The background plasma parameters at surface 4 are equal with the unperturbed plasma parameters (plasma parameters at $z=\infty$ ). The channel flow ends at the cloud surface excluding the potential drop (surface $3, z=z_{3}$ ).

For simplicity in the followings we will use for the quantities which changes along the field lines the notations: $\left(Q_{1 e}=Q_{e}\left(z=z_{1}\right), Q_{2 e}=Q_{e}\left(z=z_{2}\right) \ldots\right.$ and $\Gamma_{1 e}=\Gamma_{e}\left(z=z_{1}\right)$, $\Gamma_{2 e}=\Gamma_{e}\left(z=z_{2}\right)$ etc. $)$.

In an equilibrium plasma, far away from the pellet surface $(z=\infty)$ the energy distribution of the background plasma electrons is Maxwellian. According to [22], the energy is carried by energetic electron bunches, contributing by equal amounts to the heat transport to the pellet. This way in our model the total particle flux $\Gamma_{e}(z=\infty)$ and heat flux $Q_{e}(z=\infty)$ of the background plasma electrons is distributed among a few velocity groups (5-20), usually 5 groups. $Q_{e}(z=\infty)$ and $\Gamma_{e}(z=\infty)$ denote the single side heat and the particle flux of the random particle motion: $\Gamma_{e}(z=\infty)=\left(n_{e} / 2\right) \cdot v_{m}$, and $Q_{e}(z=\infty)=\Gamma_{e}(z=\infty) \cdot\left(2 T_{e}\right)$, where $v_{m}$ is the mean velocity $\left(k_{B}\right.$ is the Boltzmann constant, $m_{e}$ is the electron mass, and $e$ is the electron charge):

$v_{m}=\sqrt{\frac{8 k_{B} T_{e}}{\pi e m_{e}}}$.

The particle $\Gamma_{e}^{j}(z)$ and heat $Q_{e}^{j}(z)$ flux of each group are calculated as the background plasma electrons travel through the cloud by taking into account the interaction of the electron bunches with the cloud particles. The ratio of the total heat and particle fluxes give the mean energy of the particles at each toroidal cross section of the channel flow. The divergence of the heat flux is the volume heat source in the channel flow.

The spherical expansion of the cloud close to the pellet transforms into a channel flow along the field lines very smoothly [31], if the expansion is transonic. If the channel flow expansion is subsonic, 2D modeling is needed. Thus, if the cloud radius is much larger than the pellet radius the shielding effect of the spherical neutral cloud can be separated from the effect of the channel flow. Accordingly the ablation rate is calculated by Equation (1), where the electron density $n_{2 e} \sim Q_{2 e} \cdot E_{2 e}^{-1.5}$ and electron temperature $T_{2 e} \sim E_{2 e}$ corresponds to the total heat flux $\left(Q_{e}\left(z=z_{2}\right)=\Sigma Q_{e}^{j}\left(z=z_{2}\right)\right)$ and to the mean energy $\left(E_{e}\left(z=z_{2}\right)=\frac{\Sigma Q_{e}^{j}\left(z=z_{2}\right)}{\Sigma \Gamma_{e}^{j}\left(z=z_{2}\right)}\right)$ values at the spherical cloud - channel flow interface.

We would like to emphasize here that this way the shielding of the spherically expanding neutral cloud part is always considered, even if the pellet moves to the next channel leaving the field elongated part of its cloud in the previous channel. This infers the fact that the spherically expanding cloud part moves together with the pellet as it does not interact with the magnetic field. 


\section{Shielding and its effect on the ablation rate calculations}

First, the time evolution of the shield formation will be discussed, and then the average values over the time interval that the pellet spends in one flux tube $\left(t_{\text {res }}\right)$ are calculated.

The significance of the different shielding effects is analyzed through their role in the different theoretical models. Shielding effects corresponding to the different models are given in Table 1.

Along the NGS, LLP and the Hybrid code, the NGSE (Neutral Gas Shielding model including Electrostatic Shielding) model is included as well. This is an artificially created model that couples the electrostatic double shield to the NGS formula to help to understand the role of channel flow in the ablation process. The NGSE ablation rate is calculated by Equation (1), where the electron density $n_{3 e} \sim Q_{3 e} \cdot E_{3 e}^{-1.5}$ and electron temperature $T_{3 e} \sim E_{3 e}$ corresponds to the total heat flux $\left(Q_{e}\left(z=z_{3}\right)=\Sigma Q_{e}^{j}\left(z=z_{3}\right)\right)$

and to the mean energy $\left(E_{e}\left(z=z_{3}\right)=\frac{\Sigma Q_{e}^{j}\left(z=z_{3}\right)}{\Sigma \Gamma_{e}^{j}\left(z=z_{3}\right)}\right)$, behind the potential wall. The pellet radius changes in time as in the Hybrid code. In the LLP code no shielding is assumed at the beginning of the process in order to make possible comparison with and without the spherical cloud (the Hybrid and the LLP code respectively). It should be emphasized here that in the original calculations the LLP always assumes a semiempirically determined cloud part that travels with the pellet, a pre-shielding that is ignored in this study in order to highlight the importance of the spherical cloud.

\subsection{Time evolution of the shielding}

In this subsection we will analyze the effect of the electrostatic double shield and of the channel flow on the time evolution of the heat and particle flux across the pellet cloud using the Hybrid code. We will discuss first the time evolution of the electrostatic double shield during the pellet residence time $t_{r e s}$ in one flux tube. The potential wall, i.e. the electrostatic double shield forms immediately as the pellet enters the flux tube due to the considerable amount of charged particles appearing as the energy carriers start to erode the pellet surface. An electric field arises in the cloud as well, but the potential drop which corresponds to this electrostatic field is in the order of $10 \mathrm{~V}$, which is much smaller compared to the potential drop at the cloud periphery. The magnitude of the potential drop at the cloud periphery is comparable to the plasma energy content $(\gg 1000 \mathrm{~V})$, therefore the heat and particle fluxes of the background plasma electrons are considerably reduced by this potential wall. The strength of the sheath can be given by the values of the heat and particle flux ratio at the two sides of the potential wall: $Q_{3 e} / Q_{4 e}$ and $\Gamma_{3 e} / \Gamma_{4 e}$. These two values change from an initial value of 1 to about 0.3 . Figure 2a shows the above mentioned ratios for a pellet with radius $r_{p}=0.4 \mathrm{~mm}$ and velocity $v_{p}=800 \mathrm{~m} / \mathrm{s}$ ablating in a plasma with an electron density of $n_{e}=2 \cdot 10^{19} \mathrm{~m}^{-3}$ and electron temperature of $T_{e}=800 \mathrm{eV}$. Both the heat and particles flux reduction is strong at the beginning and persists during the whole residence time. On the other hand the mean energy of the particles increases due to the accelerating effect of the 
electrostatic double shielding, which is shown in Figure $2 \mathrm{a}$ as well.

In a similar way we can characterize the effect of the channel flow on the heat and particle flux with the ratios: $Q_{2 e} / Q_{3 e}, \Gamma_{2 e} / \Gamma_{3 e}$ and $E_{2 e} / E_{3 e}$. As it can be seen in Figure $2 \mathrm{~b}$, at the beginning of the ablation process in the flux tube the shielding effect of the channel flow is small and the ratios are close to unity, but as time elapses the number of particles deposited in the channel flow increases, reducing the heat flux even up to $20 \%$ of $Q_{3 e}$. As the reduction of the particle flux is stronger compared to the reduction of the heat flux, the mean energy $E_{2 e} / E_{3 e}$ of the particles increases. The energy loss function of the electrons depends on their energy, thus the slower electrons lose their energy much faster compared to the fast ones. Accordingly the mean energy of the whole electron distribution increases with time.

In the original LLP code $\left(Q_{1 e} / Q_{2 e}=1\right.$ and $\left.\Gamma_{1 e} / \Gamma_{2 e}=1\right)$, where the presence of the spherical cloud is neglected, thus it gives similar results both for the effect of the potential wall and of the channel flow.

The heat and particle flux in the cloud and the mean energy of the electrons varies in time. Accordingly the ablation rate in the Hybrid code is calculated by introducing the electron temperature $T_{2 e} \sim E_{2 e}$ and density $n_{2 e} \sim Q_{2 e} \cdot E_{2 e}^{-1.5}$ in Equation (1) at each time point. To calculate the NGSE ablation rate it is assumed that the pellet radius changes as in the Hybrid model, but the electron temperature $T_{3 e} \sim E_{3 e}$ and density $n_{3 e} \sim Q_{3 e} \cdot E_{3 e}^{-1.5}$ corresponds to the heat flux and mean energy at the end of the channel flow $\left(Q_{3 e}\right.$ and $\left.E_{3 e}\right)$. This way here we are taking into account the spherical cloud shielding and the electrostatic double shielding. Of course for the simple NGS we use $\left(Q_{4 e}\right.$ and $\left.E_{4 e}\right)$ and the original pellet radius. The ablation rate in the LLP code is considered to be the heat flux $\left(Q_{2 e}=Q_{1 e}\right)$ divided by the sublimation energy (see Equation (2)). The time evolution of the four ablation rates NGS, NGSE, LLP and Hybrid (HYB on the plots and formulas) are plotted in Figure 2c.

The NGS formula has been obtained based on a quasi-steady state model, thus the NGS ablation rate $\left(N_{N G S}^{\prime}\right)$ is constant in time if the cooling of the background plasma is neglected. The NGSE ablation rate $\left(N_{N G S E}^{\prime}\right)$ decreases as the heat flux $Q_{3 e}$ decreases, because the mean energy (and accordingly the temperature) of the particles $E_{3 e}$ is almost constant. The ablation rate calculated by the Hybrid code $\left(N_{H Y B}^{\prime}\right)$ decreases at the beginning, but as the energy increases $\left(E_{2 e}\right)$, it compensates the decrease of the heat flux $\left(Q_{2 e}\right)$ and the ablation rate increases again. The ablation rate given for an unshielded pellet $\left(N_{L L P}^{\prime}\right)$ is two orders of magnitude higher than the other three ablation rates at the beginning and is followed by a strong decrease. At the end of the local ablation process the LLP ablation rate gets close to the values given by the NGS ablation rate. On the other hand the ablation rate given by the Hybrid code can be higher compared to the NGS value.

We would like to note here, that the NGPS (Neutral Gas and Plasma Shielding) model which takes into account the shielding effect of the ionized cloud beside the shielding of the neutral cloud, gives the ablation rate as a scaling law [10]. This scaling law differs only in a constant from the NGS ablation rate. Although this model has 
been benchmarked on different machines, it did not become as widely used as the NGS scaling law [13]. However, as the NGPS scaling is very similar to the NGS scaling we will only compare our results with the NGS scaling law.

The ablation rate does not depend only on the heat flux of the electrons, but it has a strong dependence on their mean energy as well $N^{\prime} \approx Q_{e}^{0.33} \cdot E_{e}^{1.14}$. To show this dependence of the ablation rate on the heat flux and on the mean energy of the electrons, we plotted the ablation rate contours normalized to its maximum in Figure 3 . In this plot we assumed constant pellet radius.

When we consider a more complicated situation by taking into account both mean cloud parts, the ablation rate can increase in certain situations in spite of the reduction of the heat and particle flux in the channel flow due to an intense increase of mean electron energy whose exponent is the highest. The slow electrons are stopped in the channel flow far away from the pellet, and the fast, energetic electrons reach the spherical cloud surface. When all the slow electrons are trapped in the cloud, even the most energetic electrons will lose a part of their energy due to collisions with cold electrons and ions in the cloud. To underline this, we have sampled two different situations used by the Hybrid code calculations and over-plotted the time evolutions of the energy and the heat flux of the background plasma electrons reaching the spherical cloud surface for these two cases (see Figure 3). The arrows in the plot indicate how the time elapses. It can be seen that even for a large and slow pellet $\left(r_{p}=1 \mathrm{~mm}\right.$ and $v_{p}=200 \mathrm{~m} / \mathrm{s}$, $n_{e}=2 \cdot 10^{19} \mathrm{~m}^{-3}$ and $\left.T_{e}=400 \mathrm{eV}\right)$, the mean energy of the electrons increases at the beginning and it only decreases shortly before the pellet leaves the flux tube, when the channel flow shielding becomes nearly complete. For a small size and fast pellet $\left(r_{p}=0.4 \mathrm{~mm}\right.$ and $v_{p}=1000 \mathrm{~m} / \mathrm{s}, n_{e}=2 \cdot 10^{19} \mathrm{~m}^{-3}$ and $\left.T_{e}=800 \mathrm{eV}\right)$, the energy increase persists during the whole ablation process, because the channel flow remains transparent for fast electrons.

\subsection{Time averaged effect of different shielding on the ablation process}

In the previous subsection we gave the time evolution of the different shielding types in detail for a typical case. In this subsection we will calculate the average values of the same quantities for a wide range of pellet and plasma parameters during the residence time of the pellet in the flux tube. In some cases when the pellet life time was shorter than the residence time, the time interval for averaging was assumed to be the pellet life time.

The analysis was made for the following pellet and plasma parameter combinations: four pellet sizes were assumed $r_{p}=0.4,0.6,0.8,1 \mathrm{~mm}$, while the pellet velocity was assumed to be $v_{p}=200,400,600,800,1000 \mathrm{~m} / \mathrm{s}$. The background plasma density values were $n_{e}=5 \cdot 10^{18}, 10^{19}, 2 \cdot 10^{19}, 4 \cdot 10^{19}, 8 \cdot 10^{19} \mathrm{~m}^{-3}$, while the temperature values were $T_{e}=200,400,800,1600,3200 \mathrm{eV}$. The magnetic field was assumed to be $2.5 \mathrm{~T}$.

We begin our analysis by discussing the role of the potential drop $(\Phi)$. The electrostatic double shield reduces the electron particle flux drastically while the energy 
of the electrons slightly increases due to accelerating effect of this potential wall [4]. To calculate its averaged value, weighted averaging is used, where the weighting is performed according to the ablation rate. As we would like to emphasize here the particle reduction through the electrostatic double shield, we plotted the potential drop which is responsible for the electron particle flux reduction. The normalized electric potential $\Phi_{\text {norm }}=\frac{e \Phi}{T_{e}}$ is shown in Figure 4 for different electron temperatures and densities for a pellet having $1000 \mathrm{~m} / \mathrm{s}$ velocity and radius of $r_{p}=0.6 \mathrm{~mm}$. As seen in Figure 4, the potential drop is comparable to the electron temperature of the target plasma if the temperature of the plasma is higher than $200 \mathrm{eV}$ and its density is higher than $1 \cdot 10^{19} \mathrm{~m}^{-3}$, resulting in a potential barrier that reduces the particle flux to about e-th part of its original value [4]. The potential drop decreases as the density of the background plasma decreases, while its temperature dependence is influenced by the electron density: for low densities it increases with temperature and for higher densities the increase is followed by a slight decrease at very high temperatures. In the case of lower electron temperatures (less than $400 \mathrm{eV}$ ) and lower electron densities (less than $1 \cdot 10^{19} \mathrm{~m}^{-3}$ ) the value of the potential drop differs considerably from the temperature of the background plasma.

After analyzing the effect of the potential drop we will continue with the comparison of the ablation rate given by the Hybrid code against the NGSE ablation rate to evaluate how the channel flow influences the ablation rate. The ablation rate values are averaged values over the time interval that the pellet spends in the flux tube.

In order to distinguish the effect of the background plasma and pellet parameters on the time averaged ablation rate, first we assumed that the ablation process takes place in a plasma with specific parameters, and secondly we assume that the same pellet ablates in different plasmas.

Let us suppose a plasma having $T_{e}=800 \mathrm{eV}$ electron temperature and $n_{e}=$ $2 \cdot 10^{19} \mathrm{~m}^{-3}$ electron density. The Hybrid ablation rate relative to the NGSE ablation rate is shown in Figure 5. For a given pellet size $N_{H Y B}^{\prime} / N_{N G S E}^{\prime}$ increases with the pellet velocity, because faster pellets imply shorter residence time in the flux tube. During this -relatively short- time the mean energy of the background plasma electrons continuously increases resulting in an enhanced ablation rate although the heat flux decreases. On the other hand the increase of the pellet size at a given velocity will result in the reduction of Hybrid ablation rate relative to the NGSE ablation rate. It is worth noting that in the low velocity limit, the size of the very dense and low temperature cloud, which effects the ablation rate, exceeds the shielding length [29], therefore the bending of the field lines can effect the magnitude of the ablation rate. Thus, in the low velocity limit the Hybrid code can underestimate the ablation rate.

The $N_{H Y B}^{\prime} / N_{N G S E}^{\prime}$ ratio has been calculated assuming pellets with $r_{p}=0.6 \mathrm{~mm}$ and $v_{p}=1000 \mathrm{~m} / \mathrm{s}$ and plasmas having different parameters. Figure 6 shows this ratio. The Hybrid ablation rate relative to the NGSE ablation rate increases with the temperature for a given density. The slope of a curves differs for different electron densities, as the shielding effect of the channel flow depends on it. 
In the calculations presented here, it is assumed that the pellet spends a few tens of microseconds in each flux tube. The time spent there is proportional to the pellet velocity, if the pellet path is a straight line and the acceleration of the pellet is neglected. In this case the shielding effects should correlate with time, i.e. with the material deposited during the time interval the pellet spent in the flux tube. For this purpose the ratio of the Hybrid ablation rate and NGSE ablation rate is plotted as a function of the number of deposited particles for all pellet events $\left(r_{p}=0.4-1 \mathrm{~mm}\right.$ and $v_{p}=200-1000 \mathrm{~m} / \mathrm{s}$ adding pellets with $v_{p}=5000 \mathrm{~m} / \mathrm{s}$ as they deposit just a small amount of material) in Figure 7a. The density of the target plasma was $n_{e}=2 \cdot 10^{19} \mathrm{~m}^{-3}$. The symbols correspond to different temperature values. As the temperature increases, the number of deposited particles increases as well due to the fact that the ablation rate has a strong temperature dependence.

To see the effect of the spherical cloud, similar calculations were performed with the LLP code. The ablation rate given by the LLP and Hybrid code are compared in Figure $7 \mathrm{~b}$ where the $N_{H Y B}^{\prime} / N_{L L P}^{\prime}$ is shown as a function of the number of the deposited particles. As we ignored the pre-shielding in the LLP calculations, the $N_{H Y B}^{\prime} / N_{L L P}^{\prime}$ value is considerably less than unity, supporting our a priori assumption that the spherical cloud has a considerable shielding effect. To emphasize even more the effect of the neutral cloud, we compared the Hybrid ablation rate to the NGS ablation rate in Figure $7 \mathrm{c}$. The ratio of the two ablation rates is unity, if the number of particles deposited in the cloud is small $\left(N_{d e p} \approx 10^{17}\right)$. As the number of particles in the cloud start to increase the channel flow will increase the mean energy of the electrons reaching the spherical cloud. By increasing the number of deposited particles in the cloud furthermore $\left(N_{\text {dep }} \gg 10^{19}\right)$, most of the energy of the electrons will be absorbed by the cloud, thus the Hybrid ablation rate almost equals the NGS ablation rate. We would like to point out here that one of the reasons why the cloud losses its energy-filtering effect might be that we did not discretize properly the Maxwellian energy distribution, underestimating this way the ablation rate. The Hybrid ablation rate using 20 electron bunches compared to the ablation rate calculated by assuming 5 groups is shown in Figure 8. In most cases the ablation rates does not depend on the discretization of the Maxwellian. Consequently we can conclude that the NGS formula might be used if the number of deposited particles in the channel is small, but for large $\left(r_{p} \sim 1 \mathrm{~mm}\right)$ and slow $\left(v_{p} \sim 200 \mathrm{~m} / \mathrm{s}\right)$ pellets, which deposit high amount of particles, one should use detailed code calculations taking into account the presence of the ionized cloud part whose effect might be diminished by particle extraction due to grad B drifts or because of the cloud bending. Similar result were obtained by Rozhansky et al [30] who showed analytically that the total effect of the channel flow and magnetic shielding is negligible compared to the shielding of the neutral cloud. Of course this is only the case in equilibrium plasmas, because the ablation rate can be changed considerably if we take into account the effects of additional heating, non spheroid pellet shape, etc. as detailed in [28]. 


\section{Regression analysis}

The ablation rate is commonly given in a scaling form, as a power function of the electron temperature, density and pellet radius. In later models the pellet velocity has been included as well, its importance was shown in the previous subsection.

$N^{\prime}=c_{m} \cdot r_{p}^{c_{1}} \cdot v_{p}^{c_{2}} \cdot n_{e}^{c_{3}} \cdot T_{e}^{c_{4}}$

In order to find a similar formula for the ablation rate based on the Hybrid code, various plasma and pellet parameter scans were run for the typical AUG parameter regime $\left(r_{p}=0.4-1 \mathrm{~mm} ; v_{p}=200-5000 \mathrm{~m} / \mathrm{s} ; n_{e}=0.5-8 \cdot 10^{19} \mathrm{~m}^{-3} ; T_{e}: 200-3200 \mathrm{eV}\right.$; $B=2.5 \mathrm{~T}$ ). On the obtained dataset, a simple regression was performed with the scaling form set a priori to Equation (4). Here the ablation rate denotes the time averaged ablation rate in one flux tube from the Hybrid code. The results are shown in Table 2 including the original NGS exponents for easier comparison.

Comparison of the simulated ablation rates and the obtained Hybrid scaling is shown in Figure 9. As for slower pellets (indicated by red triangles in the figure) the scatter is larger, in these cases detailed calculations are encouraged instead of using the scaling.

By comparing the NGS scaling to the Hybrid scaling the exponents of the different parameters are very similar (see Table 2). The two leading parameters of both scalings are the electron temperature and the pellet radius, the importance of these parameters has already been shown in the previous subsection. The weaker dependence of the pellet radius in the Hybrid compared to the NGS model scaling possibly comes from the presence of the channel flow strongly influencing the heat and particle flux reaching the spherical cloud and in the same time reducing the role of the pellet radius in the ablation process. Regarding the electron density, the Hybrid model has a stronger dependence on this parameter, even though the channel flow is only slightly effected by the background plasma density, the electrostatic double shield strongly depends on it (Figure 4). Finally the amount of deposited material depends on the time the pellets spends in the flux tube, resulting in the obtained pellet velocity dependence neglected in earlier models.

In order to improve the scaling, three subsets of the dataset have been defined. In Subset 1, we excluded from the analyses all cases, where the ablation stopped inside the flux tube as the pellet cloud became too dense and absorbed all the energy carried by the background plasma electrons (due to the considerable change of the pellet radius). The exclusion of events, when the ablation stopped as the pellet was evaporated in a shorter time compared to the residence time, defines Subset 2. Finally Subset 3 merges the two exclusion criteria. How the scaling changes due to the different subsets is collected in Table 3. The change in case of Subset 1 is only marginal, whereas Subset 2 needs more attention. Of course if we exclude the cases where the ionized cloud exhibits a strong shielding, we get closer to the NGS model. 


\section{Comparison of experiments and the Hybrid code}

One of the goals of the codes describing the pellet ablation is to determine the penetration depth of pellets injected into plasmas with given density and temperature profiles. To compare the results obtained by the code calculations and by experiments ASDEX Upgrade discharges with stable and robust operation in the type-I ELMy Hmode have been chosen from a series of shots with similar plasma parameters, while the detailed comparison will be the subject of future work.

The simulations were done by using electron density and temperature profiles obtained by combining the discharges \#20037 and \#20113. Both the electron density and temperature profiles are recorded on the magnetic low field side of the torus therefore the density and temperature values has to be mapped on the pellet path by assuming that the density and temperature are constant on a given flux surface. The calculations were performed accordingly. As the drift effects are neglected in these calculations, the material deposition will not be discussed here.

In the above mentioned series pellets with a nominal particle content of $1.6 \cdot 10^{20}$ atoms have been injected with different velocities from the magnetic high field side $\left(v_{p}=\right.$ 240 , and $1000 \mathrm{~m} / \mathrm{s}$ with the corresponding net particle content of $9 \cdot 10^{19}, 7.2 \cdot 10^{19}$ and $3.1 \cdot 10^{19}[23]$; pellet radii corresponding to the net particle content are collected in Table 4). The penetration depths obtained by the Hybrid code were compared to the measured ones for discharges \#20041, \#20043, \#20054 [24, 25, 26].

The calculated ablation rates along the pellet path are plotted in Figure 10. The position of the separatrix is also indicated. The temperature being the leading parameter that determines ablation $\left(N^{\prime} \sim T_{e}^{1.6}\right)$ is plotted as well.

The pellet ablation monitor signal $\left(D_{\alpha}\right)$ has been collected and averaged for all pellet events in each discharge. The averaging is necessary because the pellet mass differs from pellet to pellet. The length (in time) of the pellet ablation monitor multiplied with the pellet velocity gives the penetration depth $(\lambda)$. The calculated and measured penetration depths for all analyzed shots are collected in Table 4 and they are in good agreement.

\section{Summary}

The hydrodynamical part of the LLP code describing the channel flow [20] has been modified. In the new Hybrid model the cloud formed around a pellet interacting with the hot plasma is composed of two regions. The spherical neutral cloud forms suddenly around the pellet, later on its external parts get ionized and it undergoes a channel flow along the magnetic field lines. The ablation rate has been calculated accordingly by taking into account both effects.

In this work a detailed analysis of the dependence of the ablation rate on the three main shielding effects of the pellet cloud is presented. It is assumed that the spherical cloud forming around the pellet on a sub-microsecond time scale reduces the heat flux of the target plasma considerably resulting in a relatively small ablation rate. 
The electrostatic double shield reduces the heat flux roughly to $\mathrm{e}^{\text {th }}$ part of its initial value and accordingly the ablation rate. In contrast to our expectations, the presence of the channel flow increases the ablation rate even though it reduces the heat flux reaching the spherical cloud surface. The slowest electrons are stopped in the channel flow shortly after they reach it and only the fastest electrons reach the spherical cloud. The heat flux carried by the hot electrons originating from the background plasma is consumed both by the ablation process itself and by the cloud evolution. The slowest electrons contribute mainly to cloud heating, while the fastest ones have a role in the ablation process itself. The slow and fast electrons are connected through their interaction with the cloud particles, they do not contribute independently to the ablation rate. Therefore, the Hybrid ablation rate $N_{H Y B}^{\prime}$ can exceed the NGSE ablation rate $N_{N G S E}^{\prime}$, even though the Hybrid code takes into account more "shielding" effects.

The effect of the channel flow depends mainly on the number of particles in the ionized cloud part. In most cases it drives the $N_{H Y B}^{\prime}$ above the $N_{N G S E}^{\prime}$ ablation rate. The $N_{H Y B}^{\prime} / N_{N G S E}^{\prime}$ drops below unity only for large and slow pellets. However, in such cases the ionized cloud becomes too large, therefore the cloud might be diminished by particle extraction due to drifts or because of the channel bending. For present size machines, drifts will not influence the ablation rate, just the deposition profile.

Using a simple regression fit, a compact formula for the ablation rate has been obtained similarly to former models. It has been found that the leading parameter that determines the ablation rate is the electron temperature. The fit is appropriate for fast and medium velocity pellets, while in the case of slow pellets detailed calculations are necessary.

The penetration depths obtained experimentally and numerically from the code were compared based on typical ELMy H-mode discharges. It can be concluded that reasonable agreement between the experiments and Hybrid code calculations has found. For low temperatures plasmas detailed calculation of the shielding is necessary, in contrast to higher temperature values, where the formula obtained from the linear regression is sufficient. 


\section{Acknowledgments}

The authors would like to thank S Kálvin and LL Lengyel for the useful discussions and kind support.

This work was funded by EURATOM under association contracts with Hungary and Germany. The views and opinions expressed herein do not necessarily reflect those of the European Commission.

\section{References}

[1] Milora S L et al 1995 Nucl. Fusion 35657

[2] Lang P T et al 2000 Nucl. Fusion 40245

[3] Lang P T et al 1996 Nucl. Fusion 361531

[4] Lengyel L L et al 1996 Nucl. Fusion 361679

[5] Veres G and Lengyel L L 1997 J. Nucl. Mat. 25096

[6] Rozhansky V et al 1995 Plasma Phys. Control. Fusion 37399

[7] Rozhansky V et al 2004 Plasma Phys. Control. Fusion 46575

[8] Parks P B 1996 Plasma Phys. Control. Fusion 38571

[9] Parks P B and Turnbull J R 1978 Phys. Fluids, 211735

[10] Pégourié B et al 1993 Nucl. Fusion 33591

[11] Kaufmann M et al 1986 Nucl. Fusion 26171

[12] MacAulay A K et al 1994 Nucl. Fusion 3443

[13] Garzotti L et al 1997 Nucl. Fusion 371167

[14] Ishizaki R et al 2004 Phys. Plasmas 114064

[15] Parks P B and Rosenbluth M N 1998 Phys. Plasmas, 51380

[16] Samulyak R et al 2007 Nucl. Fusion 47103

[17] Durst R Det al 1990 Nucl. Fusion 303

[18] Wurden G A et al 1990 Rev. Sci. Inst. 613604

[19] Lengyel L Let al 1994 Nucl. Fusion 34675

[20] Lengyel L L et al 1999 Nucl. Fusion 39791

[21] Parks P B 1996 Plasma Phys. Control. Fusion 38571

[22] Houlberg W A et al 1988 Nucl. Fusion 28595

[23] Lang P T et al Rev. Sci. Instrum. 74 (2003) 3974.

[24] Kálvin S et al 2005 Proc. of the 32nd EPS Conf. on Controlled Fusion and Plasma Physics (Tarragona) vol 29C (European Physical Society, 2005) P-2.078

[25] Lang P T et al 2005 Proc. of the 32nd EPS Conf. on Controlled Fusion and Plasma Physics (Tarragona) vol 29C (European Physical Society, 2005) O-4.003

[26] Gál K et al 2005 Proc. of the 32nd EPS Conf. on Controlled Fusion and Plasma Physics (Tarragona) vol 29C (European Physical Society, 2005) P-2.098

[27] Mcneill D H 1985 Phys. Rev. Lett 551398

[28] Pegourier B 2007 Plasma Phys. Control. Fusion 49 R87

[29] Parks P B 1992 Nucl. Fusion 322137

[30] Rozhansky V and Senichenkov I 2005 Plasma Phys. Reports 31, 993

[31] Kálvin S et al 2003 Proc. of the 30nd EPS Conf. on Controlled Fusion and Plasma Physics (St. Petersburg) vol 27A (European Physical Society, 2003) P-3.154 


\section{Tables}

\begin{tabular}{l|c|c|c|c}
\hline Name & NGS & NGSE & LLP & HYBRID \\
\hline spherical cloud & $\mathrm{X}$ & $\mathrm{X}$ & - & $\mathrm{X}$ \\
channel flow & - & - & $\mathrm{X}$ & $\mathrm{X}$ \\
electrostatic double shield & - & $\mathrm{X}$ & $\mathrm{X}$ & $\mathrm{X}$ \\
\hline
\end{tabular}

Table 1. Models and shielding types

\begin{tabular}{l|l|l|l|l|l}
\hline & $c_{m}$ & $c_{1}$ & $c_{2}$ & $c_{3}$ & $c_{4}$ \\
& const. & radius & velocity & density & temperature \\
\hline Hybrid & $2.56 \cdot 10^{16}$ & 1.03 & 0.18 & 0.42 & 1.58 \\
NGS & $3.78 \cdot 10^{16}$ & 1.33 & 0. & 0.33 & 1.64 \\
\hline
\end{tabular}

Table 2. Exponents of the parameters in the Hybrid code based scaling regression and in the NGS formulae

\begin{tabular}{l|l|l|l|l}
\hline & $c_{1}$ & $c_{2}$ & $c_{3}$ & $c_{4}$ \\
& radius & velocity & density & temperature \\
\hline Hybrid & 1.03 & 0.18 & 0.42 & 1.58 \\
Subset 1 & 1.07 & 0.20 & 0.41 & 1.53 \\
Subset 2 & 1.11 & 0.09 & 0.37 & 1.57 \\
Subset 3 & 1.15 & 0.11 & 0.37 & 1.54 \\
\hline
\end{tabular}

Table 3. Powers of the parameters in the Hybrid code based for different data subsets

\begin{tabular}{l|l|l|l|l}
\hline Shot & $v_{p}[\mathrm{~m} / \mathrm{s}]$ & $r_{p}[\mathrm{~mm}]$ & Meas. $\lambda[\mathrm{cm}]$ & Calc. $\lambda[\mathrm{cm}]$ \\
\hline 20041 & 240 & 9.1 & $\sim 17$ & $\sim 15$ \\
20043 & 600 & 7.2 & $\sim 21$ & $\sim 21$ \\
20054 & 1000 & 0.5 & $\sim 18$ & $\sim 19$ \\
\hline
\end{tabular}

Table 4. Comparison of the calculations with the measurements 


\section{Figures}

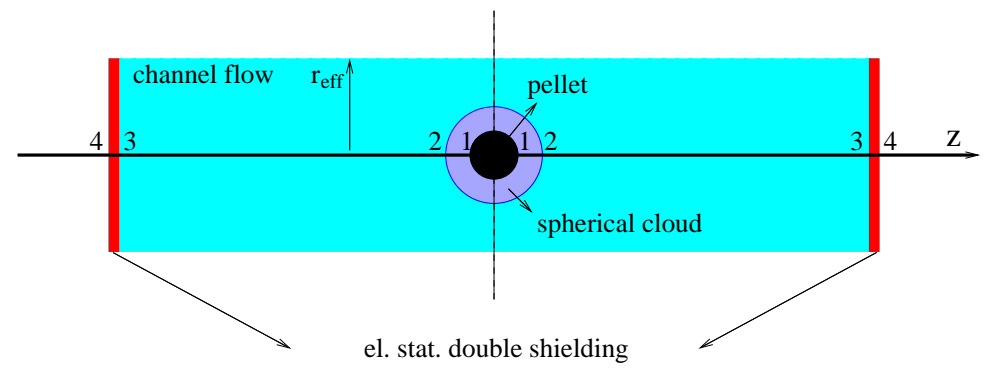

Figure 1. The structure of the shielding clouds surrounding the pellet. The numbers indicate various surfaces: 1-pellet surface, 2-spherical cloud surface, 3-channel flow surface, 4-cloud surface. $r_{\text {eff }}$ is the effective radius of the flux tube. 


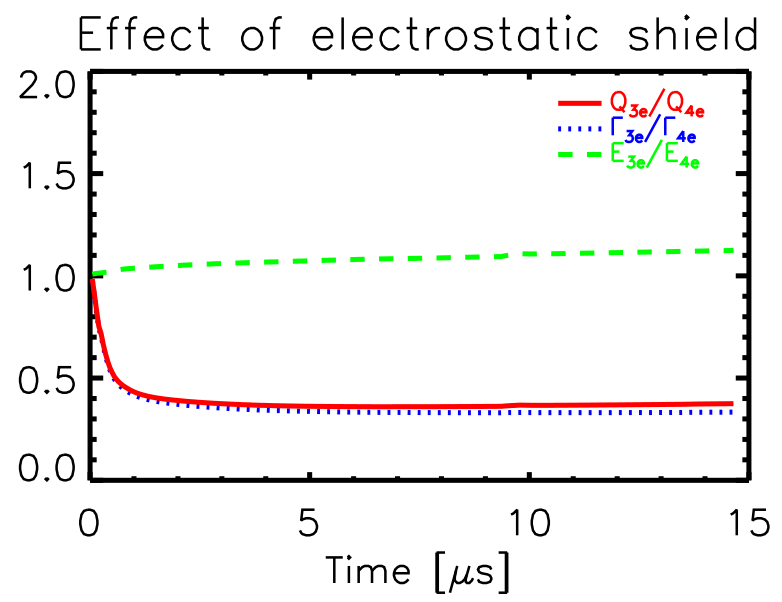

(a)

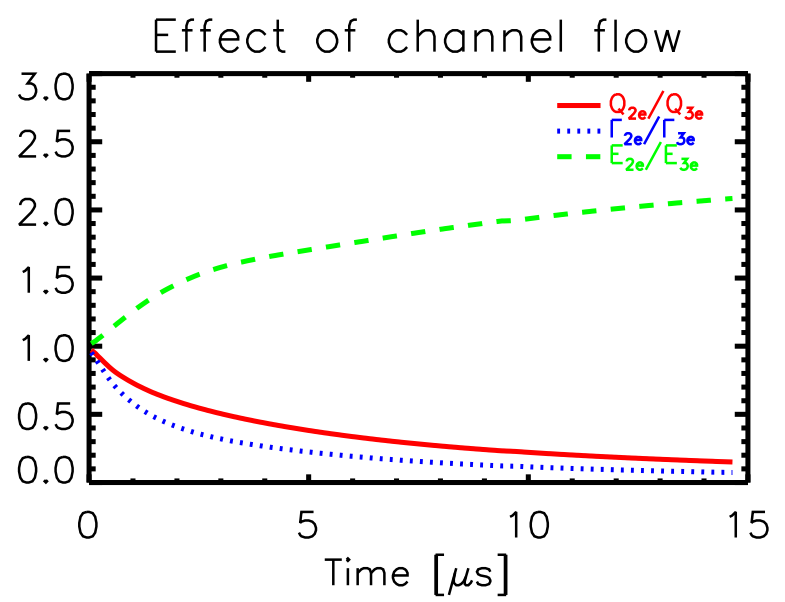

(b)

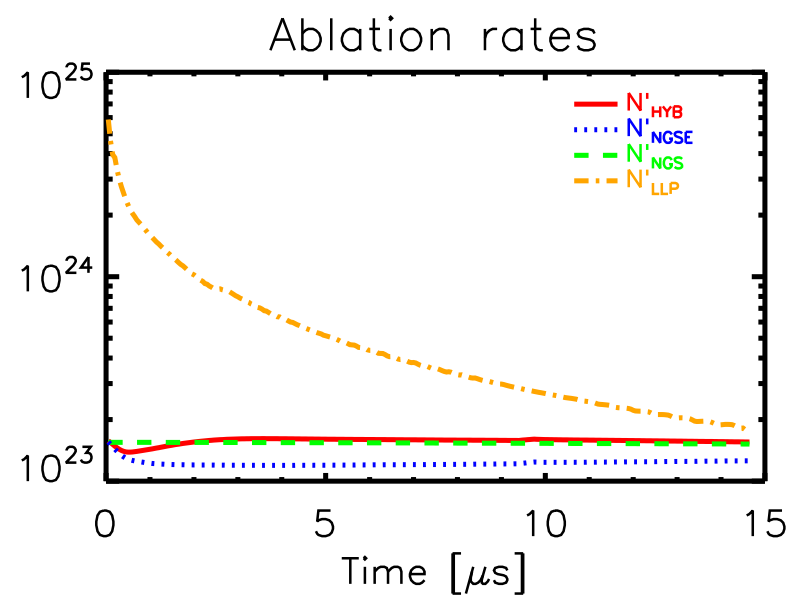

(c)

Figure 2. Time evolution of the ratio of the heat and particle flux and the mean energy at the two ends of the channel flow (a) and across the potential drop (b) for a pellet with radius $r_{p}=0.4 \mathrm{~mm}$ and velocity $v_{p}=800 \mathrm{~m} / \mathrm{s}$ calculated by the Hybrid code for a plasma with an electron density of $n_{e}=2 \cdot 10^{19} \mathrm{~m}^{-3}$ and electron temperature of $T_{e}=800 \mathrm{eV}$. The time evolution of the ablation rate calculated by the Hybrid model, the NGSE formula, LLP code, and NGS formula for the same parameters. 


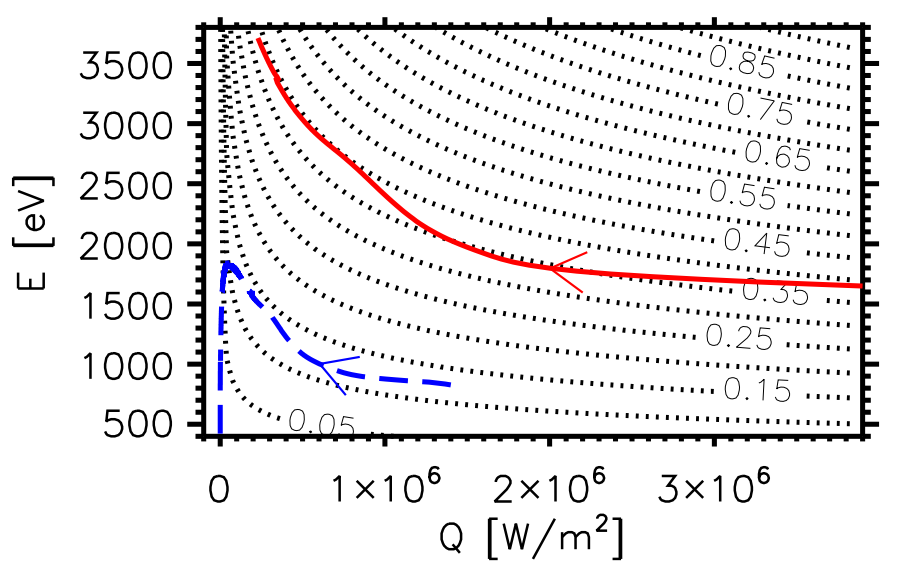

Figure 3. NGS ablation rate contours - dotted lines - as a function of the heat flux and of the mean electron energy of the background plasma. The time evolution of the background plasma mean electron energy and heat flux which reach the neutral cloud (surface 2 in Figure 1) for two distinct pellet events: $r_{p}=1 \mathrm{~mm}, v_{p}=200 \mathrm{~m} / \mathrm{s}$, $n_{e}=2 \cdot 10^{19} \mathrm{~m}^{-3}$ and $T_{e}=400 \mathrm{eV}$ (dashed line); and $r_{p}=0.4 \mathrm{~mm} ; v_{p}=1000 \mathrm{~m} / \mathrm{s}$, $n_{e}=2 \cdot 10^{19} \mathrm{~m}^{-3}$ and $T_{e}=800 \mathrm{eV}$ (continuous line). 


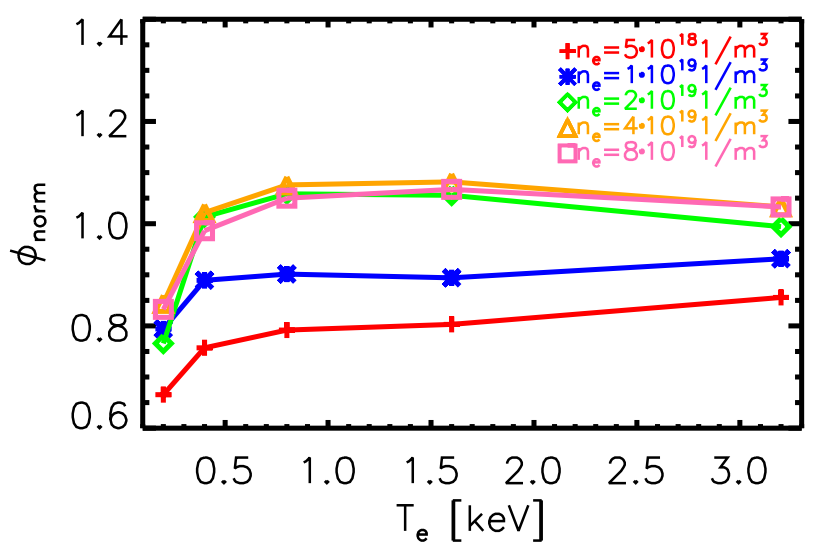

Figure 4. The normalized potential drop as a function of the temperature of the target plasma for pellets having $1000 \mathrm{~m} / \mathrm{s}$ velocity and $r_{p}=0.6 \mathrm{~mm}$ radius and densities as it is indicated on the figure. 


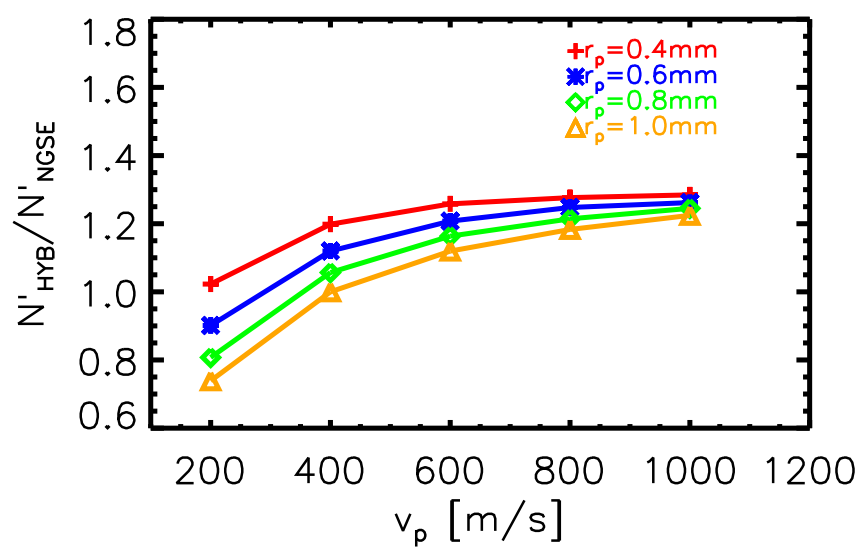

Figure 5. The ratio of the Hybrid ablation rate and the NGSE ablation rate as a function of velocity is plotted for four pellet sizes: $r_{p}=0.4 \mathrm{~mm}$ (plus), $r_{p}=0.6 \mathrm{~mm}$ (star), $r_{p}=0.8 \mathrm{~mm}$ (rhomb), $r_{p}=1 \mathrm{~mm}$ (triangle). The target plasma temperature is $800 \mathrm{eV}$ while its density is $2 \cdot 10^{19} \mathrm{~m}^{-3}$. 


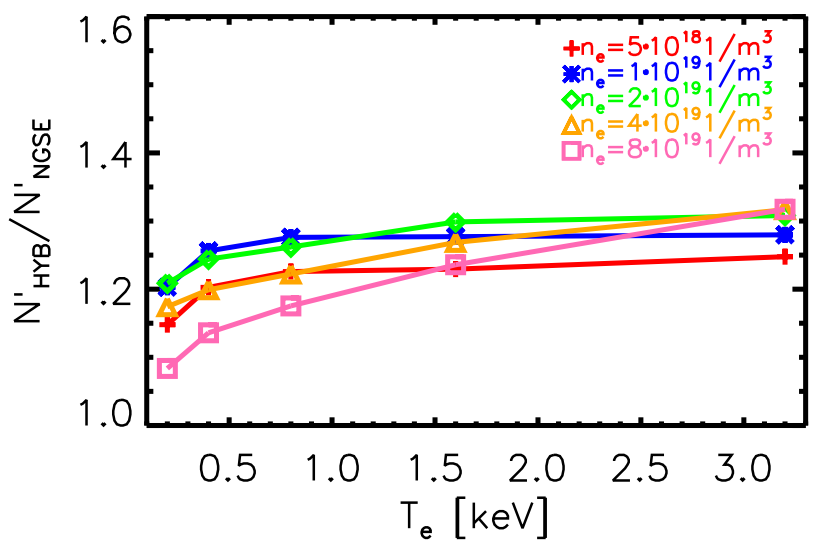

Figure 6. The ratio of the Hybrid ablation rate and the NGSE ablation rate as a function of temperature is plotted for five values of the target plasma electron density: $n_{e}=5 \cdot 10^{18} \mathrm{~m}^{-3}$ (plus), $n_{e}=10^{19} \mathrm{~m}^{-3}$ (star), $n_{e}=2 \cdot 10^{19} \mathrm{~m}^{-3}$ (rhomb), $n_{e}=4 \cdot 10^{19} \mathrm{~m}^{-3}$ (triangle) and $n_{e}=8 \cdot 10^{19} \mathrm{~m}^{-3}$ (square). The pellet characteristics are: radius: $r_{p}=0.6 \mathrm{~mm}$ and velocity $v_{p}=1000 \mathrm{~m} / \mathrm{s}$. 


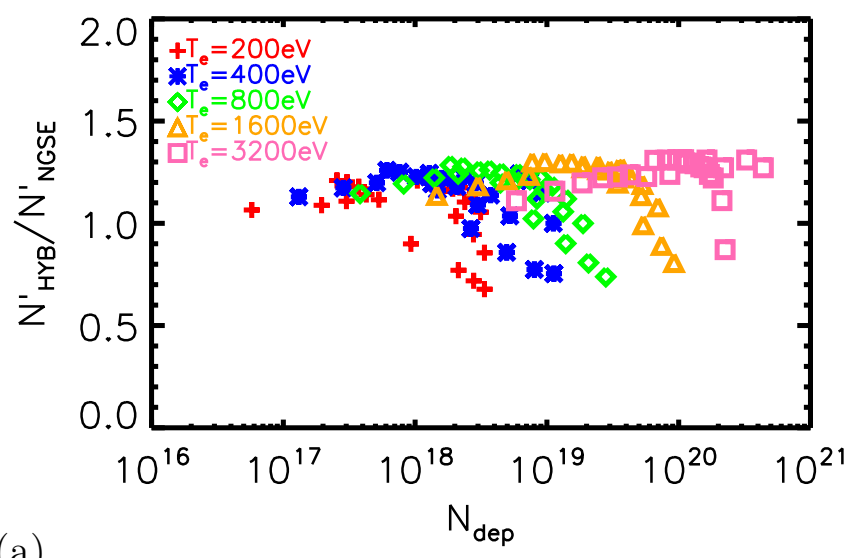

(a)
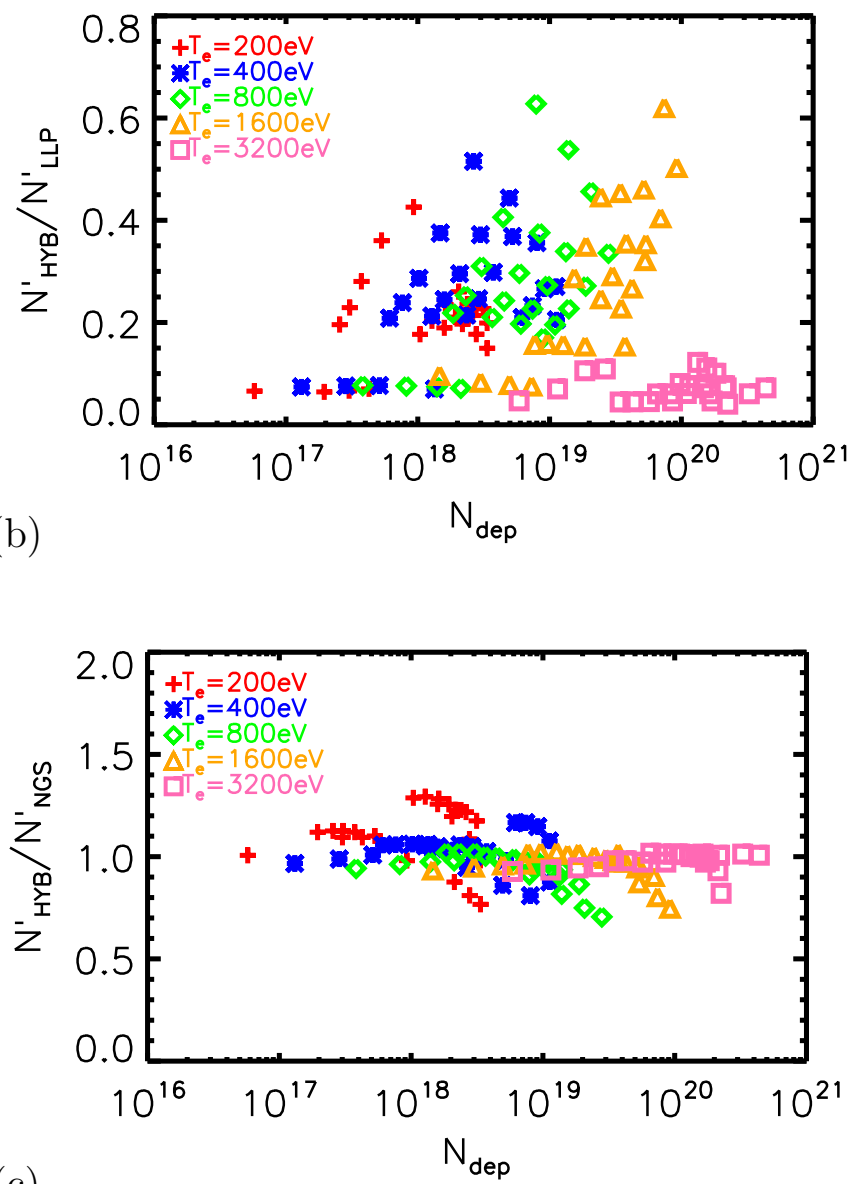

(c)

Figure 7. The ratio of the Hybrid ablation rate and the NGSE ablation rate (a), the ratio of the Hybrid ablation rate and the LLP ablation rate (b) and the ratio of the Hybrid ablation rate and the NGS ablation rate (c) as a function of the number of deposited particles for different target plasma temperatures. In all cases the target plasma density is $n_{e}=2 \cdot 10^{19} \mathrm{~m}^{-3}$ and the data correspond to 4 pellet radii, $r_{p}=0.4-1 \mathrm{~mm}$ and 6 pellet velocities, $v_{p}=200-5000 \mathrm{~m} / \mathrm{s}$. 


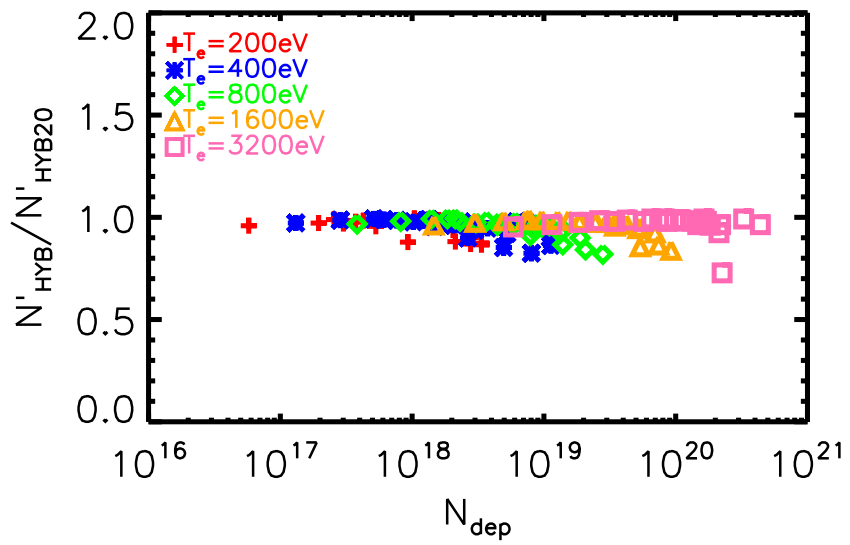

Figure 8. The ratio of the Hybrid ablation rate with 5 energy groups and the Hybrid ablation rate using 20 energy groups as a function of the number of deposited particles for different target plasma temperatures. The target plasma density is $n_{e}=2 \cdot 10^{19} \mathrm{~m}^{-3}$ and the data correspond to 4 pellet radii, $r_{p}=0.4-1 \mathrm{~mm}$ and 6 pellet velocities, $v_{p}=200-5000 \mathrm{~m} / \mathrm{s}$. 


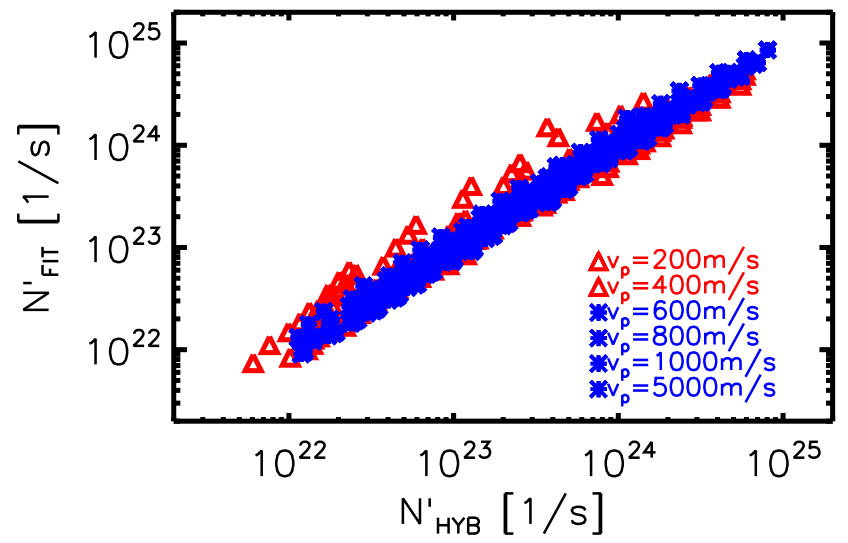

Figure 9. The ablation rate calculated from the regression formula versus the ablation rate from the Hybrid code calculations. 


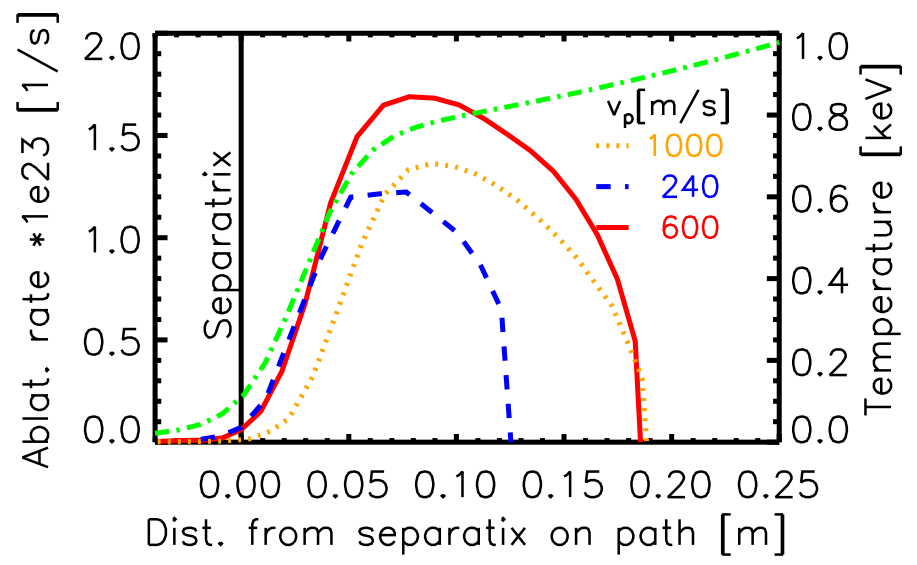

Figure 10. The calculated ablation rates and the corresponding plasma electron temperature (dashed-dotted green) on the pellet path as a function of the distance from the separatrix for shot \#20041 dashed blue (velocity $240 \mathrm{~m} / \mathrm{s}$ ), \#20043 continouos red (velocity $600 \mathrm{~m} / \mathrm{s}$ ), \#20054 dotted orange (velocity $1000 \mathrm{~m} / \mathrm{s}$ ) 